\title{
Magnetic resonance imaging in clinically isolated lesions of the brain stem
}

\author{
IEC ORMEROD, A BRONSTEIN, P RUDGE, G JOHNSON, D MACMANUS, \\ AM HALLIDAY, H BARRATT, EPGH DU BOULAY, BE KENDAL, IF MOSELEY, \\ SJ JONES, A KRISS, E PERINGER
}

From the National Hospital for Nervous Diseases and the Institute of Neurology, Queen Square, London, UK

SUMMARY Twenty-seven patients with an isolated brain stem syndrome, thought to be due to demyelination, were examined by magnetic resonance imaging (MRI). A brain stem lesion was identified in 25 , and clinically silent lesions outside the brain stem were demonstrated in 20 . MRI was more sensitive than evoked potentials in detecting brain stem and other lesions. The scan findings were compared with those in 23 patients with multiple sclerosis, who had chronic brain stem dysfunction, with particular reference to the distribution of abnormalities and the MRI characteristics of the lesions. The relaxation times, $T_{1}$ and $T_{2}$, of the lesions were measured by MRI. These values were seen to fall in serial studies of acute lesions, but remained unchanged in the chronic lesions. MRI may therefore allow the age of lesions to be assessed.

Disturbance of brain stem function is frequently found in patients with multiple sclerosis and may be the presenting symptom. ${ }^{1}$ However, it is not known what proportion of patients presenting with an isolated episode of brain stem dysfunction will ultimately prove to have multiple sclerosis. Evoked potentials and $x$ ray computed tomography (CT) have been of limited value in detecting evidence of multiple lesions and examination of the cerebrospinal fluid (CSF) does not consistently show an immunological abnormality in the central nervous system (CNS) of such patients. Since MRI is a sensitive method for detecting plaques in the CNS in patients with known multiple sclerosis, ${ }^{2}$ we have studied a number of patients presenting with signs of brain stem dysfunction and compared the abnormalities found on MRI with those in patients who have clinically definite multiple sclerosis and a similar brain stem disturbance.

The aim of the study was fourfold. Firstly, to determine the sensitivity of MRI in identifying lesions

Address for reprint requests: Dr IEC Ormerod, University Department of Clinical Neurology, The National Hospital for Nervous Diseases, Queen Square, London WCIN 3BG, UK

Received 3 September 1985.

Accepted 16 October 1985 within the brain stem. Secondly, to compare the sites of the lesions visualised by MRI with the site of damage suggested by neurological and neuro-otological examination. Thirdly, to establish the incidence of clinically unsuspected lesions in parts of the brain other than the brain stem detected by MRI, and to compare these findings with the results of evoked potential studies and the examination of the CSF. Fourthly, to study the natural history of the brain stem lesions with particular reference to the changes in MRI parameters.

\section{Methods}

\section{Subjects}

Thirty patients (aged 17 years to 49 years, mean 27.9 years), in whom there was evidence of isolated brain stem dysfunction, were examined. None had a history of neurological dysfunction prior to the development of brain stem symptoms. Subjects aged over 50 years and those with evidence of vascular disease were excluded. In all patients a diagnosis of multiple sclerosis was thought to be possible by the referring neurologist. In addition, 23 patients (aged 24 years to 58 years, mean 36 years) with clinically definite multiple sclerosis were scanned. All had long-standing evidence of brain stem dysfunction. A third group of $\mathbf{5 0}$ neurologically normal control subjects (aged 18 years to 60 years, mean 42 years) was also studied. Examinations performed were approved by the Ethics Committee of The National Hospitals, Queen Square and Maida Vale and conformed to the guidelines of The National Radiological Protection Board. 
Table 1 The abnormalities of evoked potentials and CSF in patients with clinically isolated brain stem lesions. The patients were divided on the basis of the duration of their symptoms. Group I with symptoms for less than 3 months and Group II with symptoms for 9 months or more

\begin{tabular}{lllll}
\hline & VEP & AEP & SEP & CSF oligoclonal \\
\hline Group I & $(N=17)$ & $(N=17)$ & $(N=16)$ & $(N=15)$ \\
Normal & 14 & 14 & 14 & 9 \\
Abnormal & 3 & 3 & 2 & 6 \\
Group II & $(\mathrm{N}=10)$ & $(\mathrm{N}=9)$ & $(\mathrm{N}=8)$ & $(\mathrm{N}=7)$ \\
Normal & 3 & 6 & 6 & 3 \\
Abnormal & 7 & 3 & 2 & 3 \\
\hline
\end{tabular}

\section{$2 M R I$}

All the patients studied underwent MRI examination. This was performed with a $0.5 \mathrm{~T}$ Picker imaging system (a few initial studies were done at $0.25 \mathrm{~T}$ ), studying protons. Multislice imaging protocols were used with inversion recovery (IR) and spin echo (SE) sequences for the axial scans and additional sagittal slices were made in most patients. In many individuals computer reformatted images of the relaxation time constants, $T_{1}$ and $T_{2}$, were made and measurements of these values were taken from both normal brain stem and from the lesions. The scans were assessed blind by a panel of independent assessors.

\section{Neurological assessment}

All patients were examined clinically including assessment of eye movements, gait and balance. In addition, a full neurootological assessment was carried out in those patients with isolated brain stem lesions and in those with definite multiple sclerosis who had brain stem lesions. The latter comprised: (a) DC ENG recording from each eye. Saccadic velocities were determined for $30^{\circ}$ movements in the horizontal plane. Pursuit was studied in the horizontal plane for target displacements of $30^{\circ}$ at frequencies of 0.1 to $2 \mathrm{~Hz}$. Nystagmus was sought in the light and dark in the primary position of gaze and on displacement of the eye $30^{\circ}$ to the left and right in the horizontal plane or vertically. (b) Opto-kinetic nystagmus was assessed using a small rotating drum in the horizontal and vertical planes and a full field stimulation in the horizontal plane. (c) Vestibular function was assessed with binaural, bi-thermal caloric testing, sinusoidal oscillations and impulsive rotation in the horizontal plane. The effect of fixation was determined for all three methods of inducing the vestibulo-ocular reflex. (d) Positional nystagmus was elicited by the Hallpike manoeuvre. (e) Pure tone audiometry and stapedius reflex recording (both ipsi- and contralaterally).

\section{Evoked potential examination}

Visual (VEP), auditory (AEP), and median and posterior tibial somatosensory (SEP) evoked potentials were recorded in the majority of subjects in the patient groups (clinically isolated lesions of the brain stem and clinically definite multiple sclerosis with brain stem dysfunction). The examinations were performed without prior knowledge of the MRI findings and the methods conformed to those previously described. ${ }^{3}$

\section{CSF}

In the majority of subjects in the patient groups the CSF was examined for the presence of oligoclonal bands.

\section{Results}

\section{MRI, CSF and evoked potentials}

Thirty patients were examined in whom the diagnosis of a clinically isolated brain stem lesion was made on the basis of a combination of long tract and brain stem signs, the presence of nystagmus of central origin or an internuclear ophthalmoplegia. Although the diagnosis of demyelination was raised in all patients of this group at presentation, three patients were proved to have an alternative diagnosis: one brain stem glioma, one brain stem angioma and one pontine haemorrhage. In the remaining 27 patients the age range was 21 to 49 years (mean 31.5 years), and the duration of symptoms was 1 week to 12 years (mean 74 weeks). These 27 fell into two clear subgroups in terms of the duration of their symptoms; group I, with symptoms for less than 3 months and group II, with symptoms in excess of 9 months.

Table 2 The incidence and distribution of MRI abnormalities in patients with multiple sclerosis and those with a clinically isolated presentation (Group I, II)

\begin{tabular}{|c|c|c|c|c|c|c|}
\hline & Brain stem & Cerebellar & Only $P$ & Only DC & $P+D C$ & $\begin{array}{l}\text { Any cerebral } \\
\text { abnormalities }\end{array}$ \\
\hline $\begin{array}{l}\text { Group I }(N=17) \\
\text { Group II }(N=10) \\
\text { Multiple sclerosis }(N=23)\end{array}$ & $\begin{array}{l}15 \\
10 \\
21\end{array}$ & $\begin{array}{l}2 \\
2 \\
8\end{array}$ & $\begin{array}{l}4 \\
1 \\
2\end{array}$ & $\begin{array}{l}1 \\
\mathbf{0} \\
\mathbf{0}\end{array}$ & $\begin{array}{r}8 \\
6 \\
21 \\
\end{array}$ & $\begin{array}{r}13 \\
7 \\
23 \\
\end{array}$ \\
\hline
\end{tabular}

$\mathrm{P}=$ Periventricular lesions.

$\mathrm{DC}=$ Discrete cerebral white matter lesions. 


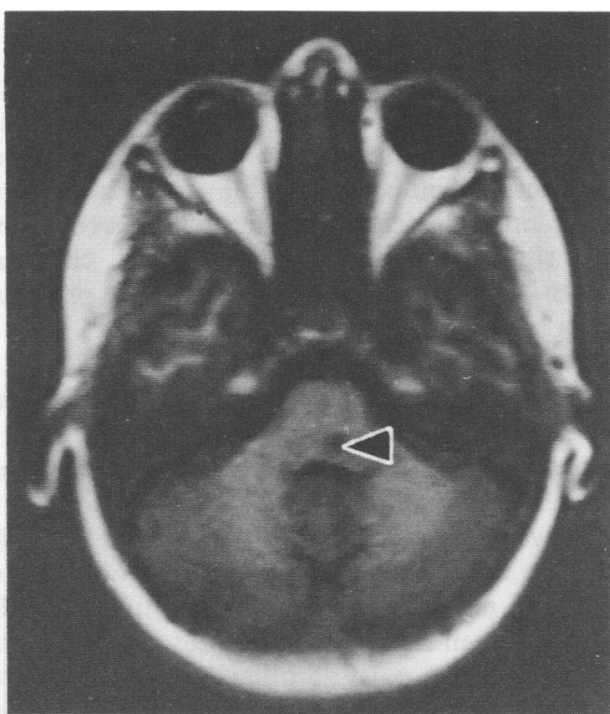

Fig 1 An inversion recovery image in a patient with an isolated VI nerve palsy. The lesion (arrowed) is deeply sited, presumably involving the VI nerve fibres.

Group I There were 17 patients within this group; the age range was 21 to 48 years (mean 29.8 years) and the duration of symptoms was between one week and 3 months (mean 3.6 weeks). Full evoked potential studies were performed in all but one patient who did not have SEPs. CSF was examined in all but two patients. The VEPs were abnormal in three patients indicating a lesion remote from the brain stem and an

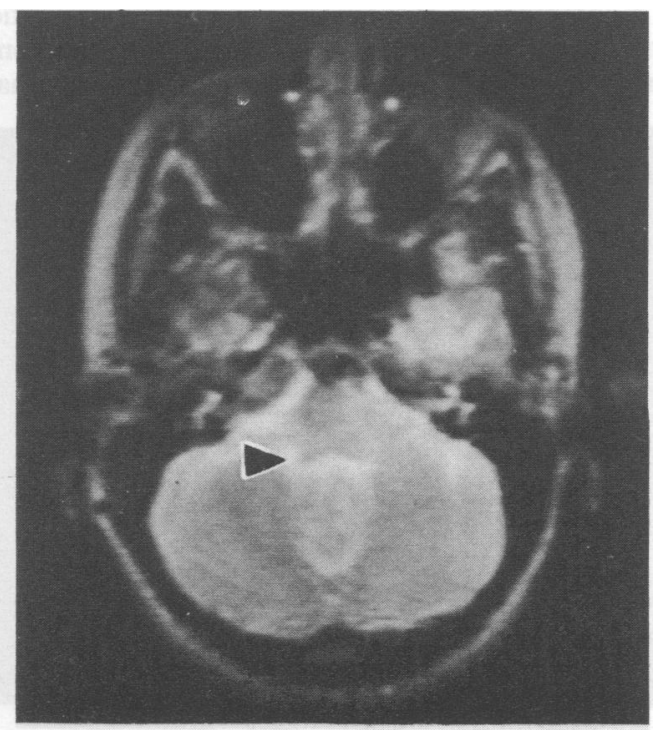

Fig 2 A spin echo image in a patient with a VII nerve palsy. The laterally placed lesion (arrowed) is presumed to have damaged the VII nerve fibres.
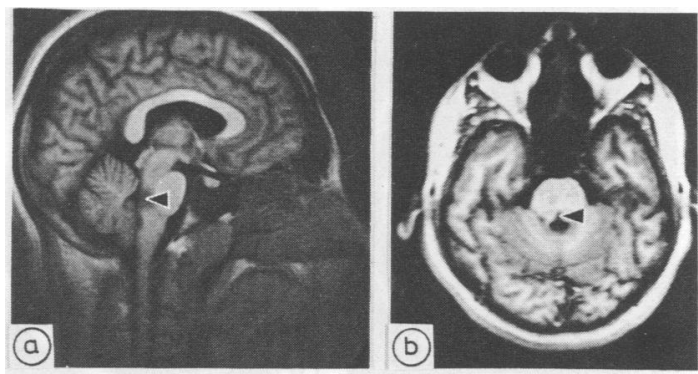

Fig 3 Inversion recovery images. The sagittal (a), and axial (b) cuts demonstrate the lesion (arrowed) extending into the pons from the floor of the fourth ventricle. The patient had bilateral VII palsies and a horizontal gaze palsy.

oligoclonal pattern was found in the CSF in six patients (table 1). Only three patients had an abnormal AEP and in two the SEP was abnormal; these abnormalities could have been due to a single brain stem lesion and did not help to clarify the diagnosis. Two of the 17 patients in this group had normal MRI scans of the brain stem; one of these had evidence of periventricular lesions in the supra-tentorial sections. Neither of these two patients had abnormal evoked potentials. In all, 13 of the 17 patients showed abnormalities outside the brain stem (table 2). In the two patients with a single lesion in the brain stem but no other lesions supra-tentorially detected by MRI, one had an oligoclonal CSF and one an abnormal VEP. Group II This group comprised 10 patients (aged 22 to 49 years, mean 31.8 years) in whom demyelination of the brain stem was suspected although symptoms had been present for 9 months or longer. The duration of brain stem symptoms ranged from 9 months to 12 years (mean 46.5 months). All the patients had evidence of brain stem lesions on their scans (table 2). Eight patients had a full set of evoked potential studies; in four either the AEP, the SEP, or both indicated a lesion in the brain stem (table 1). In seven patients MRI showed lesions outside the brain

(a)

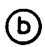

Right eye

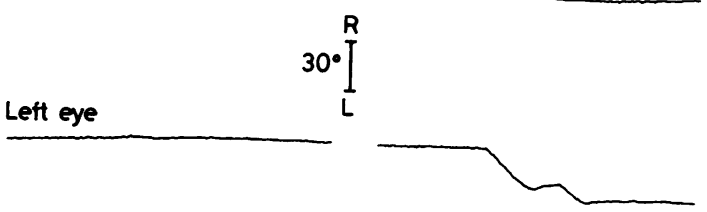

Fig 4 ENG in a patient with bilateral internuclear ophthalmoplegia (the MRI is shown in Figure 5). There is failure of adduction of both left $(A)$ and right $(B)$ eyes. 

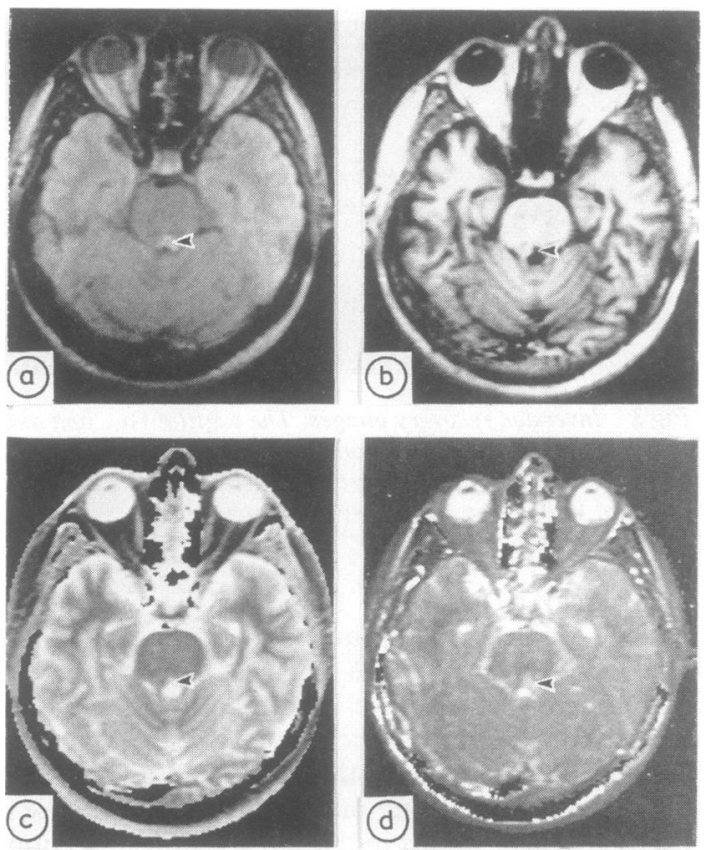

Fig 5 Axial scans in a patient with a bilateral internuclear ophthalmoplegia. The lesion is seen on the floor of the fourth ventricle on the spin echo (a), inversion recovery $(b), T_{1}(c)$ and $T_{2}(d)$ images.

stem and in six of these the VEPs were also abnormal. In one patient where the MRI failed to reveal additional, subclinical, abnormalities the VEP was abnormal. In seven patients the CSF was examined and oligoclonal bands were demonstrated in three, all of whom had MRI evidence of cerebral lesions.

Normal control subjects Of the 50 subjects in this group, three had abnormalities on MRI; in two there were a small number of discrete lesions within the cerebral white matter; in the third there were widespread lesions indistinguishable from those seen in definite multiple sclerosis. There was nevertheless a highly significant difference ( $p<0.001$, chi square) between the cerebral MRI changes in patients of groups I and II and the controls.

\section{Correlation of site of the MRI lesion in the brain stem and physical signs for groups I and II}

There was a good correlation between the presence of a VI nerve palsy and the lesion seen on the MRI scan which was either in the floor of the 4th ventricle or deeper in the pons, presumably involving the nucleus of the VI nerve or its fibres respectively (fig 1). A lesion was seen in one of these sites in all six patients with a VI nerve palsy. Similarly, in all of the eight patients with VII nerve palsies lesions were seen at an appropriate site within the brain stem, including the three with bilateral VII palsies. In the cases with clinical evidence of an isolated VII palsy the MRI lesions were more lateral in the region where the VII nerve fibres pass laterally (fig 2).

Horizontal gaze palsies were present in three patients and in each case lesions were visualised in an area of the brain stem which could have included the paramedian pontine reticular formation (PPRF), an area thought to be responsible for the generation of saccades.

A combination of these three clinical abnormalities was not infrequently seen. Three patients had combined VI and VII nerve lesions. Another patient had
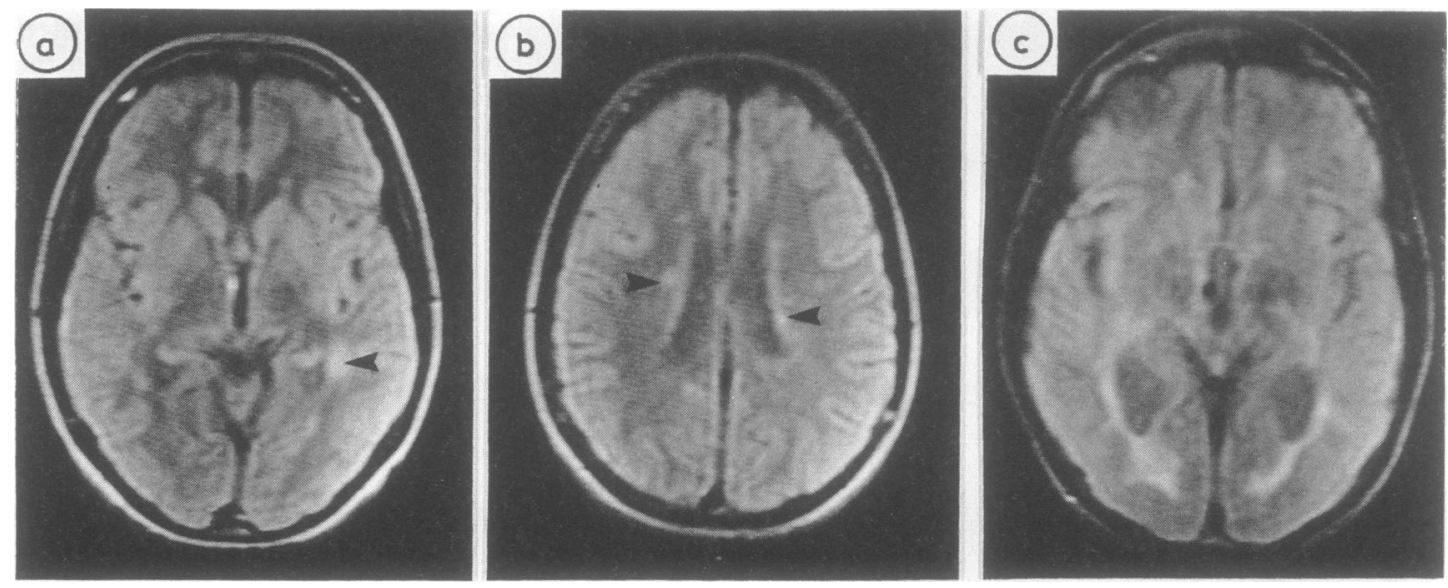

Fig 6 Axial spin echo images, at 2 levels through the lateral ventricles, showing clinically unsuspected lesions (arrowed) in a patient with an acute clinical brain stem lesion $(a, b)$. Comparable sections (c) in a patient with clinically definite multiple sclerosis show similar but more extensive lesions. 


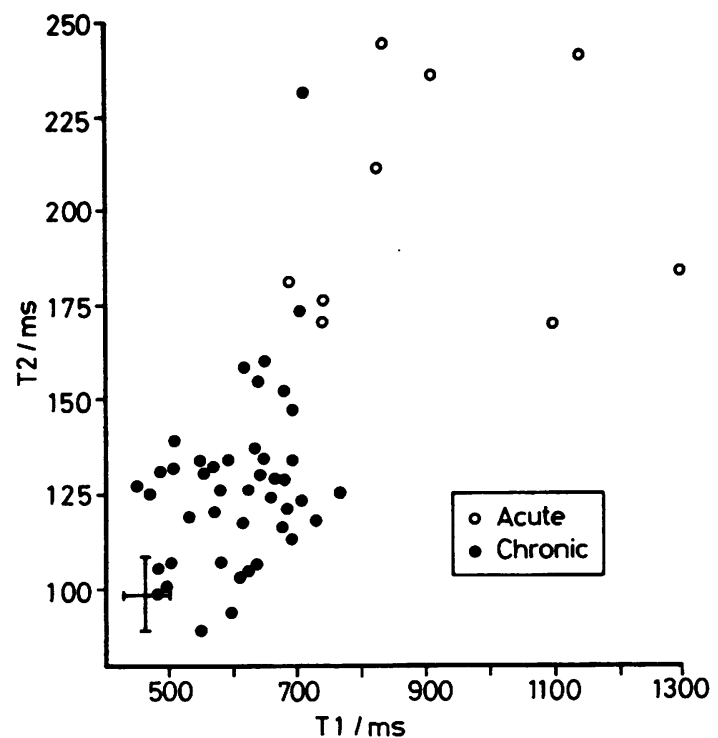

Fig 7 A scatter plot of $T_{1}$ versus $T_{2}$ for acute and chronic brain stem lesions. There is little overlap between the two groups. The mean and standard deviations are shown of $T_{1}$ and $T_{2}$ for normal brain stem from the control subjects.

a complete horizontal gaze palsy together with bilateral VII palsies; the MRI lesion was midline, extend-

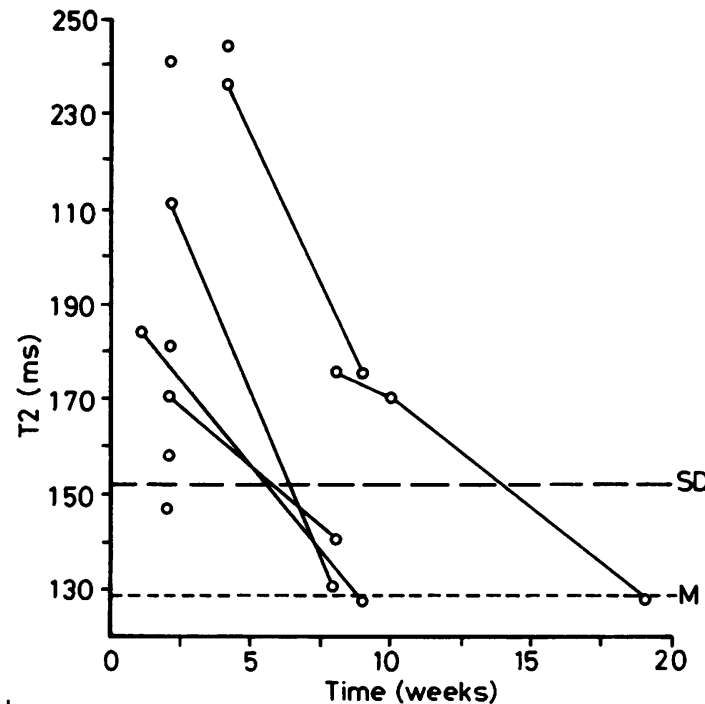

Fig 8 A plot of $T_{1}$ of acute brain stem lesions against time from the onset of brain stem symptoms. Serial measurements from the same lesion are joined together. The mean (M) and standard deviation (SD) of the $T_{1}$ values of the chronic lesions are shown for comparison. ing from the floor of the IV ventricle into the pons (fig 3 ), presumably involving the VII nerve fibres at the genu, as well as the PPRF or the part of the VI nerve nucleus concerned with conjugate horizontal gaze.

An internuclear ophthalmoplegia was seen in seven cases five of which were bilateral (fig 4) and the area of the medial longitudinal fasciculus (MLF) was involved in five cases (fig 5). However in one patient with an internuclear ophthalmoplegia no lesion was apparent in the brain stem on MRI and in another there were so many lesions that no anatomical localisation was possible.

Four of the six patients with upbeat nystagmus had a lesion in the floor of the IV ventricle, a site known to be associated with this abnormality; ${ }^{4}$ in two other patients with this sign, there was a solitary lesion in the medulla in one and no lesion was apparent in the other. All nine patients with abnormalities of the stapedius reflex had pontine lesions. There was no consistent site of lesions in patients who had pursuit defects, including unilateral pursuit abnormality, or caloric dysfunction.

\section{Comparison of lesions seen on MRI in Groups I} and II and patients with definite multiple sclerosis There was remarkable similarity in the distribution of the brain stem lesions between the patients who presented with clinically isolated lesions and the patients with multiple sclerosis. The floor of the IV ventricle and cerebral aqueduct were the most fre-

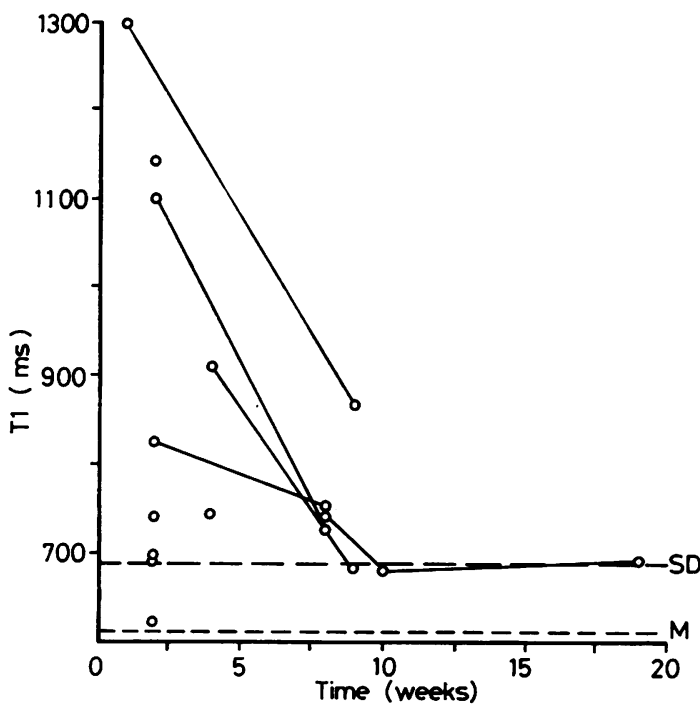

Fig 9 A plot of $T_{2}$ of acute brain stem lesions against time from the onset of brain stem symptoms. Serial measurements from the same lesion are joined together. The mean (M) and standard deviation (SD) of the $T_{2}$ values of the chronic lesions are shown for comparison. 
quent sites for the lesions. Of the 25 patients with MRI evidence of clinically isolated brain stem lesions, $10 \mathrm{had}$ a lesion in one of these sites compared with 18 of 21 patients in the group of clinically definite multiple sclerosis. In the supra-tentorial cuts changes were again most frequently seen around the ventricles in both the patients with clinically isolated lesions and with clinically definite multiple sclerosis. Periventricular changes were seen in all 23 patients with multiple sclerosis and in 19 of the $27(70 \%)$ patients with clinically isolated brain stem lesions (table 2). These changes were of similar distribution in these two groups although the abnormalities seen in patients presenting with clinically isolated lesions were less extensive than those seen in patients with established multiple sclerosis (fig 6).

\section{Evolution of the brain stem lesions}

Contrast in the MR images is multi-parametric and is mainly dependent on the proton density and the relaxation times $T_{1}$ and $T_{2} \cdot{ }^{5}$ We therefore reformatted the images to produce computed images of $T_{1}$ and $T_{2}$, as observations of intensity alone would not allow the contribution of these parameters to the signal intensity to be assessed. Measurements taken of the brain stem lesions showed that the acute lesions usually had elevated $T_{1}$ and $T_{2}$ values relative to those seen in the chronic brain stem lesions (fig 7), which were in turn higher than those recorded from the normal brain stem in our control group. On follow-up examination the MR characteristics of the acute lesions were seen to fall towards those seen in the chronic lesions (figs 8,9 ). $T_{1}$ and $T_{2}$ of the chronic lesions showed no significant change over a 3 month period.

\section{Discussion}

Until the early 1970s methods of diagnosing multiple sclerosis depended upon the clinical demonstration of two or more lesions in the central nervous system in a patient with symptoms for 1 year or more, in whom there was a relapsing and remitting course and where no other cause was found to account for their disability. ${ }^{6}$ Since then evoked potential methods have been developed which may demonstrate clinically silent lesions and most neurologists now utilise these methods to assist in the diagnosis of multiple sclerosis. The presence of oligoclonal bands in the CSF is characteristic of multiple sclerosis and some diagnostic classifications ${ }^{7}$ include these and evoked potential abnormalities in assessing the certainty of the diagnosis. However, neither the evoked potential abnormalities, nor those of the CSF are specific to multiple sclerosis. Imaging of the cerebral hemispheres and brain stem, by conventional CT scanning, has been disappointing in multiple sclerosis even using high dose contrast enhancement in an attempt to localise lesions where the blood brain barrier is defective. ${ }^{8}$ MRI was first reported to be of value in detecting plaques in the brain in $1981,{ }^{89}$ an observation which has been confirmed by other groups ${ }^{210}$ and this study.

Of more clinical concern, however, is the patient who presents with an isolated lesion in his first episode. Patients presenting with optic neuritis have a high chance of developing multiple sclerosis during their lifetime and many studies have followed large groups of such patients. ${ }^{11}$ On the other hand, patients presenting with isolated brain stem lesions have not been followed systematically to determine whether they subsequently develop the disease. The few follow-up data that are available suggest that patients, for example with VI nerve palsies, have a small risk of subsequently developing multiple sclerosis. ${ }^{12}$

In this study we found a high proportion of our patients have MRI changes in the brain similar to those seen in established multiple sclerosis. It is important to note that our patients were highly selected and cannot be taken as representative of the general population developing isolated cranial nerve palsies, in that they have already been referred to a neurological institute because a neurologist thought the lesion was most likely to be central. This automatically excludes a large number of patients presenting to a general physician with, for example, VI or VII nerve palsies or nystagmus. Be that as it may, in this study evoked potential studies were of limited value in detecting remote lesions in patients presenting acutely although rather better in those who have had their disorder for a number of years. CSF examination was abnormal in a higher proportion of acute lesions but the presence of oligoclonal bands is not specific to multiple sclerosis. In contrast to this, MRI detected multiple lesions in the brain in over $70 \%$ of patients and demonstrated the lesion in the brain stem in all but three cases. The question arises as to the specificity of these lesions. Although lesions of similar appearance can be seen in patients with vascular disease, especially over the age of 50 years, ${ }^{13}$ we tentatively suggest that in the present study multiple sclerosis is the most likely diagnosis. In comparing the scans of the patients with clinically isolated brain stem lesions and those with established multiple sclerosis there are striking similarities. In particular the periventricular lesions which are so characteristic of the disease from pathological studies ${ }^{14}$ were seen in both groups of patients, the occipital horns and trigones being most frequently involved. Similarly, the discrete white matter lesions were indistinguishable. These findings support the 
concept that many of the patients with apparently isolated lesions have multiple sclerosis. Proof must await long term follow-up.

If, as seems probable, an estimate of the age of lesions can be made from their $T_{1}$ and $T_{2}$ characteristics, it may be possible to determine the time-course of the cerebral and brain stem abnormalities, and determine whether all lesions are accrued simultaneously in a patient. The origin of the increased $T_{1}$ and $T_{2}$ of the lesions is not fully established. Oedema probably contributes in the acute phase and resolution of oedema and inflammation is the presumed mechanism of the fall in $T_{1}$ and $T_{2}$ to values seen in chronic lesions where replacement of myelin by glial fibres is the most prominent histological change. There is evidence that the protons of myelin do not contribute to the signal obtained from normal brain $^{1314}$ and the MR characteristics of chronic lesions are thought to be due to an increase in water, compared with normal white matter, and an altered physicochemical environment.

The prognostic implications of the cerebral abnormalities seen in patients with isolated lesions are not, as yet, determined and it is not possible to make a diagnosis of multiple sclerosis on the basis of disseminated lesions on MRI in a patient with a single clinical episode. If multiple lesions of different ages were demonstrated in a patient there would be grounds for assuming that the neurological illness was not monophasic. Such a finding would suggest a disease which was still active and in which lesions were accumulated over a longer period of time, a process known to occur in multiple sclerosis. The diagnosis of multiple sclerosis remains essentially clinical and demyelinating lesions within the CNS without clinical sequelae are not sufficient grounds on which to base the diagnosis. Long term follow-up relating MRI changes to eventual clinical outcome will reveal the significance of such abnormalities. Our results are part of a prospective study which we hope will resolve these unanswered problems.

The MRI facility was established by the Multiple Sclerosis Society and is also supported by the Medical Research Council. We thank Margaret Henry for her secretarial assistance.

\section{References}

${ }^{1}$ Matthews WB, Acheson ED, Batchelor JR, Weller RO. McAlpine's Multiple Sclerosis. Edinburgh, Churchill Livingstone, 1985.

${ }^{2}$ Runge VM, Price AC, Kirshner HS, Allen JH, Partain CL, James AE. Magnetic resonance imagine of multiple sclerosis: a study of pulse-technique efficiency. $A J R$ 1984;143:1015-26.

${ }^{3}$ Halliday AM, ed. Evoked Potentials in Clinical Testing. Edinburgh, Churchill Livingstone, 1982.

${ }^{4}$ Fisher A, Gresty M, Chambers B, et al. Primary position upbeating nystagmus. Brain 1985;106:949-64.

${ }^{5}$ Young IR, Bailes DR, Burl M, et al. Initial clinical evaluation of a whole body nuclear magnetic resonance (N.M.R.) tomography. J Comp Assist Tomog. 1982;6:1-18.

${ }^{6}$ McDonald WI, Halliday AM. Diagnosis and classification of multiple sclerosis. Br Med Bull 1977;33:4-8.

${ }^{7}$ Poser CM, Paty DW, Scheinberg L, et al. New diagnostic criteria for multiple sclerosis: guide lines for research protocols. Ann Neurol 1983;13:227-31.

${ }^{8}$ Vinuela FV, Fox AJ, Debrun GM; Feasby TE, Ebers GC. New perspectives in computed tomography of multiple sclerosis. AJR 1982;139:123-7.

${ }^{9}$ Young IR, Pallis CA, Bydder GM, Hall AS, Legg NJ, Steiner RE. Nuclear magnetic resonance imaging in multiple sclerosis. Lancet 1981;ii:1063-6.

${ }^{10}$ Lukes SA, Crooks LE, Aminoff MJ, et al. Nuclear magnetic resonance imaging in multiple sclerosis. Ann Neurol 1983;13:592-601.

${ }^{11}$ Hutchinson WM. Acute optic neuritis and the prognosis for multiple sclerosis. J Neurol Neurosurg Psychiatry 1976;39:283-9.

${ }^{12}$ Rush JA, Younge BR. Paralysis of cranial nerves III, IV and VI. Arch Ophthalmol 1981;99:76-9.

${ }^{13}$ Ormerod IEC, Roberts RC, du Boulay EPGH, et al. NMR in multiple sclerosis and cerebral vascular disease. Lancet 1984;ii: 1334.

${ }^{14}$ Lumsden CE. The neuropathology of multiple sclerosis. In: Vinken PJ, Bruyn GW, eds. Handbook of Clinical Neurology, vol 9. Amsterdam: North Holland, 217-9.

${ }^{15}$ Bottomley PA, Hart HR, Edelstein WA, et al. Normal human brain studied by magnetic resonance at 1.5 Tesla. Radiology 1984;150:441-6.

${ }^{16}$ Pykett IL, Rosen B. Nuclear magnetic resonance: in vivo proton chemical shift imaging. Radiology 1983; 149:197-201. 